\title{
Quark model calculations of transition form factors at high photon virtualities
}

\author{
G. Ramalho ${ }^{1}$ \\ ${ }^{1}$ Laboratório de Física Teórica e Computacional - LFTC, Universidade Cruzeiro do Sul/Universidade Cidade de São Paulo, \\ 01506-000, São Paulo, SP, Brazil
}

\begin{abstract}
We present calculations of $\gamma^{*} N \rightarrow N^{*}$ transition form factors, where $N$ is the nucleon and $N^{*}$ is a nucleon resonance, based on a covariant quark model. Our main focus is at high photon virtualities (large $Q^{2}$ ) where the valence quark degrees of freedom dominate the contributions to the transition form factors and helicity amplitudes. In that regime, the quark model estimates can be compared with the available data, particularly with the Jefferson Lab data at intermediate and large momentum transfer $\left(Q^{2}>2 \mathrm{GeV}^{2}\right)$. The main focus is on the $\Delta(1232) 3 / 2^{+}, N(1440) 1 / 2^{+}, N(1535) 1 / 2^{-}$and $N(1520) 3 / 2^{-}$resonances, but estimates for other higher mass resonances are also discussed.
\end{abstract}

\section{Introduction}

In the last two decades important information about the electromagnetic structure of the nucleon and the nucleon resonances have been collected in modern facilities such as Jefferson Lab (JLab), MAMI, MIT-Bates and ELSA, among others [1-5]. Helicity amplitudes and form factors associated with the $\gamma^{*} N \rightarrow N^{*}$ transitions have been measured for resonances $N^{*}$ with masses up to $2 \mathrm{GeV}$ for values of $Q^{2}$ in the range $Q^{2}=0-8 \mathrm{GeV}^{2}[1,6]$, where $Q^{2}=-q^{2}$, and $q$ is the momentum transfer. With the JLab$12 \mathrm{GeV}$ upgrade, we expect to probe even larger values of $Q^{2}[1,5]$.

To interpret the new data above $Q^{2}=2 \mathrm{GeV}^{2}$, theoretical models based on relativity are fundamental. Models which include valence quark degrees of freedom are preferable, since those are the expected dominant degrees of freedom at large $Q^{2}$ [1-4]. These models can be used to make predictions for transition form factors at large $Q^{2}$, and may also be used to guide future experiments.

In the present work, we discuss mainly estimates from the covariant spectator quark model $[5,7,8]$. The covariant spectator quark model is a model based on constituent quarks where the quark electromagnetic structure is parametrized in order to describe the nucleon electromagnetic structure. The wave functions of the baryons are ruled by the spin-flavor-radial symmetries [9], with radial wave functions determined phenomenologically with the assistance of empirical data, lattice data or estimates of the quark core contributions $[1,5]$. One can then use parametrizations of a few resonances $N^{*}$ to make predictions for other states based on the symmetries. The model is covariant by construction and can then be used in applications at large $Q^{2}$. In some cases the model can be extended with the inclusion of effective descriptions of the meson cloud effects, which can have significant contributions at small $Q^{2}$ [10-19].
In the next section we describe the formalism associated with the covariant spectator quark model. In Sect. 3, we present estimates for $\gamma^{*} N \rightarrow N^{*}$ transition form factors at large $Q^{2}$ for the states: $\Delta(1232), N(1440), N(1520)$, $N(1535), N(1880), N(1700)$ and $\Delta(1700)$. In Sect. 4 we present estimates for the $\Delta(1232)$ quadrupole form factors at low $Q^{2}$. Our summary and conclusions are presented in Sect. 5 .

\section{Covariant spectator quark model}

The covariant spectator quark model $[5,7,8]$ is based on the three main ingredients:

1. the baryon wave function (including the nucleon) is represented in terms of the spin-flavor structure of the individual quarks based on the $S U_{S}(2) \times S U_{F}(3)$ spin-flavor symmetry, rearranged as an active quark and a spectator quark-pair [7, 8, 20];

2. within the impulse approximation, the three-quark system is reduced to a quark-diquark system, parametrized by a radial wave function $\psi_{B}$, after the integration into the quark-pair degrees of freedom $[7,8,20]$;

3. the electromagnetic structure of the quark is parametrized by quark isoscalar/isovector and strange quark form factors $f_{i \pm}\left(Q^{2}\right)$ and $f_{i s}\left(Q^{2}\right)(i=1$ for Dirac, and $i=2$ for Pauli), which simulate the substructure associated with the gluons and quarkantiquark effects, represented according to the vector meson mechanism [7, 8, 12, 21].

When the nucleon wave function $\left(\Psi_{N}\right)$ and the resonance wave function $\left(\Psi_{R}\right)$ are both expressed in terms of 
the single quark and quark-pair states, the transition current in impulse approximation can be written as $[7,8,20]$

$$
J^{\mu}=3 \sum_{\Gamma} \int_{k} \bar{\Psi}_{R}\left(P_{R}, k\right) j_{q}^{\mu} \Psi_{N}\left(P_{N}, k\right),
$$

where $P_{R}, P_{N}$, and $k$ are the resonance, the nucleon, and the diquark momenta, respectively. In the previous equation the index $\Gamma$ labels the intermediate diquark polarization states, the factor 3 takes account of the contributions from the other quark pairs by the symmetry, and the integration symbol represents the covariant integration over the diquark on-mass-shell momentum. In the study of the inelastic transitions, we use the Landau prescription to ensure the current conservation [14, 22, 23].

Using Eq. (1), we can express the transition current in terms of the quark electromagnetic form factors $f_{i \pm}$ $(i=1,2)$ and the radial wave functions $\psi_{N}$ and $\psi_{R}$ for resonances with no strange quarks and determine the transition form factors $[7,14,23,24]$. Taking advantage of the quark form factor structure, based on vector meson dominance, the model has been extended to the lattice QCD regime (heavy pions and reduced meson cloud) $[8,12,21]$, to the nuclear medium $[25,26]$ and to the timelike regime $\left(Q^{2}<0\right)[15,17,27,28]$.

The present description of the covariant spectator quark model takes into account only the effects associated with the valence quark degrees of freedom. There are however some processes, such as the meson exchanged between the different quarks inside the baryon, which cannot be reduced to processes associated with the dressing of a single quark. Those processes can be regarded as a consequence of the meson exchanged between the different quarks inside the baryon, and can be classified as meson cloud corrections to the hadronic reactions $[14,15,18,19]$.

The study of the role of the meson cloud effects on the $\gamma^{*} N \rightarrow N^{*}$ transition can be done also in the context of the dynamical coupled-channel reaction models (dynamical models) [4, 29-32]. Those models use baryonmeson states to describe the photo- and electro-production of mesons by nucleons, taking into account the meson dressing of propagators and vertices, and are fitted to the data. The bare core contribution to the data can be determined by removing the effect of the meson-baryon dressing of propagators and vertices [31]. Those estimates of the bare core can be used to test the limits of models based on valence quarks or even to calibrate parameters of the quark models.

The separation of the transition form factors into the valence quark and meson cloud contributions is model dependent [33,34], and the identification of the bare baryon states depends of the calibration of the background $[4,32]$. One can, however, reduce the impact of the model dependence comparing our estimates of the valence quark contributions with lattice QCD simulations for large pion masses. Although quenched and unquenched lattice QCD simulations include some meson cloud effects, those effects are reduced for large pion masses $[35,36]$.

To compare our model with the lattice QCD simulations, we use our calibration of the radial wave functions for the baryon lattice QCD masses and the quark current, $j_{q}^{\mu}$, expressed in terms of the lattice QCD vector meson masses $[8,12,21]$. The free parameters of the radial wave functions and quark current are the same in the physical and lattice QCD regimes. The extrapolation of the results for the physical limit is obtained when we replace the dependence on the lattice QCD masses by the physical masses.

\section{Transition form factors at large $Q^{2}$}

We present next our estimates for the transition form factors for the $\Delta(1232)$, radial excitations of the nucleon and some negative parity states, including the states $N(1520)$ and $N(1535)$.

We use $M_{N}$ and $M_{R}$ to represent the nucleon and the resonance masses, respectively. In the case of the $\Delta(1232)$, we use $M_{\Delta}$, exceptionally.

\section{1 $\gamma^{*} N \rightarrow \Delta(1232)$ transition}

The $\gamma^{*} N \rightarrow \Delta(1232)$ transition is known to be dominated by the magnetic transition $[1,37]$, as a consequence of a quark spin flip in the nucleon to form a $\Delta(1232)$ with spin $\frac{3}{2}\left(\Delta^{+}\right.$or $\left.\Delta^{0}\right)$. Quark models, however, underestimate the magnitude of the magnetic form factor, $G_{M}^{*}$, near $Q^{2}=0$. This property can be naturally explained in the context of the covariant spectator quark model, when we consider that the nucleon and the $\Delta(1232)$ systems are mainly composed by $s$-wave quark-diquark states [10]. In that case, one concludes, based on the normalization of the radial wave functions and the Cauchy-Schwarz inequality for integrals that $G_{M}^{*}(0) \leq 2.07$, well below the experimental value $G_{M}^{*}(0) \simeq 3[5,10,18]$.

The missing strength in quark model estimates of $G_{M}^{*}$ can be understood when we consider the effect of the pion cloud dressing in addition to the valence quark effects. Dynamical models estimate that the contribution of the pion cloud effects can be about $30-45 \%$ of $G_{M}^{*}$ at low $Q^{2}[4,29-$ 31]. The natural conclusion is that quark models cannot describe the $\gamma^{*} N \rightarrow \Delta(1232)$ completely, and therefore the $\Delta(1232)$ radial wave function $\psi_{\Delta}$ cannot be extracted directly from the data $[5,10,12]$.

One needs, then to rely on indirect methods to estimate $\psi_{\Delta}$. There are two main options: i) use some independent estimate of the contribution of the valence quark effects to $G_{M}^{*}$; ii) use lattice QCD simulations of the form factors to determine the function $\psi_{\Delta}$, assuming that the pion cloud effects are small due to the large pion masses.

The first option can be implemented using the EBAC/Argonne-Osaka [31] estimate for the bare core contributions, obtained when the baryon-meson couplings are turned off $[30,31]$. In this limit, we obtain the blue points from Fig. 1 in the left and central panels. One can see that those points (bare contribution) are well described by the fits used to determine $\psi_{\Delta}$ (dashed lines in the left and central panels). At large $Q^{2}$, we can see in the central panel of Fig. 1, that the valence quark contributions are dominant and describe well the data.

As mentioned, $\psi_{\Delta}$, can also be estimated by a fit of the model parameters to a lattice QCD data with 

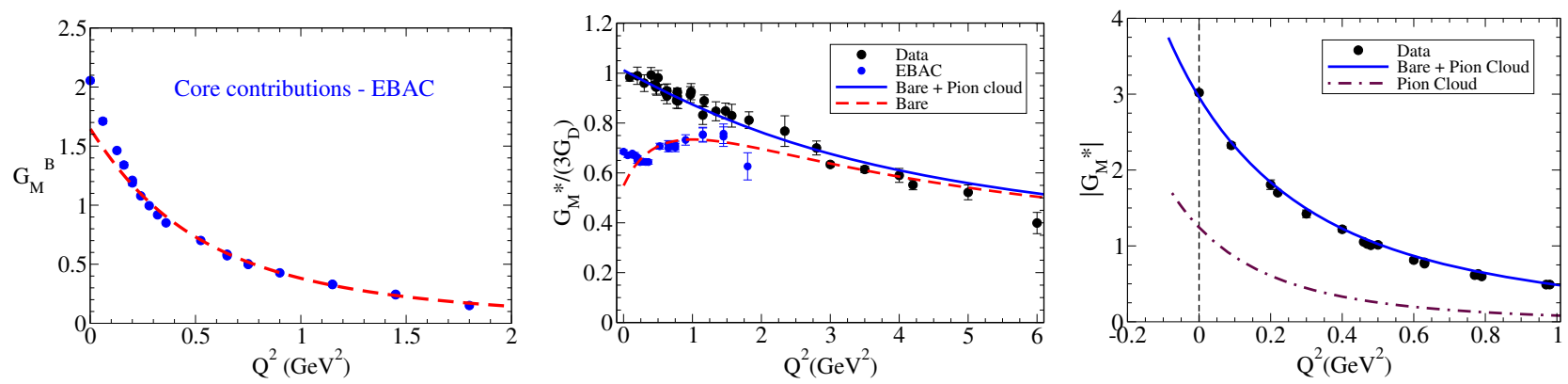

Figure 1. $\gamma^{*} N \rightarrow \Delta(1232)$ magnetic form factor. Left: Comparison of bare contribution with EBAC estimate from [31]. Center: Form factor normalized by $3 G_{D}$, where $G_{D}=\left(1+Q^{2} / \Lambda_{D}^{2}\right)^{-2}$, with $\Lambda_{D}^{2}=0.71 \mathrm{GeV}^{2}$. Right: Re-parametrization of $\left|G_{M}^{*}\right|$ based on expression (2) for the pion cloud with $\Lambda_{D}^{2}=0.9 \mathrm{GeV}^{2}$ [28]. Extension to the timelike region. Database from [10, 11].

$m_{\pi} \simeq 400-600 \mathrm{MeV}[12,36]$. In this case, the pion cloud effects are reduced and one obtains a cleaner estimate of the valence quark effects. Based on the extension of the model to the lattice QCD regime [12, 21], we conclude that the parametrization derived from the EBAC estimate is also consistent with the lattice QCD data from [36], as shown in [12]. The main conclusion is then that $\psi_{\Delta}$ can be determined either by lattice QCD data or by the EBAC estimate of the bare core.

To describe the $G_{M}^{*}$ data, we need to include some estimate of the pion cloud effects $\left(G_{M}^{\pi}\right)$. Since our framework is based on valence quarks, we choose to simulate the pion cloud effects by a simple parametrization: $G_{M}^{\pi}=3 \lambda_{\pi} G_{D}\left(\frac{\Lambda_{\pi}^{2}}{\Lambda_{\pi}^{2}+Q^{2}}\right)^{2}$, where $\lambda_{\pi} \simeq 0.448$ and $\Lambda_{\pi}^{2} \simeq 1.53$ $\mathrm{GeV}^{2}$ [11]. The final result for $G_{M}^{*}$ is represented by the solid line in the central and right panels of Fig. 1. In the more recent applications of the model, which include the $\gamma^{*} N \rightarrow \Delta(1232)$ transition in the timelike region [27, 28], we consider an alternative parametrization based on the connection with the microscopic pion cloud mechanisms $[18,28]$. We consider in particular, the parametrization

$$
\begin{aligned}
G_{M}^{\pi}= & \frac{3}{2} \lambda_{\pi} F_{\pi}\left(q^{2}\right)\left(\frac{\Lambda_{\pi}^{2}}{\Lambda_{\pi}^{2}-q^{2}}\right)^{2}+ \\
& \frac{3}{2} \lambda_{\pi} \frac{\Lambda_{D}^{4}}{\left(\Lambda_{D}^{2}-q^{2}\right)^{2}+\Lambda_{D}^{2}\left[\Gamma_{D}\left(q^{2}\right)\right]^{2}},
\end{aligned}
$$

where the first term is associated with the photon-pion coupling, proportional to the pion electromagnetic form factor $F_{\pi}\left(q^{2}\right)$, and the second term is associated with the photon coupling with the intermediate baryon states. This last coupling is simulated by an effective dipole function $G_{D}$ with $\Lambda_{D}^{2}=0.90 \mathrm{GeV}^{2}$, and include an effective width $\Gamma_{D}$ in order to avoid singularities in the region $q^{2}=-Q^{2}>0$. The new parametrization is presented in the right panel of Fig. 1 (dashed-dotted line). The advantage of the last parametrization of $G_{M}^{\pi}$ is that the model can be naturally extended to the timelike region, above $Q^{2}=-\left(M_{\Delta}-M_{N}\right)^{2}$. These estimates are useful for the study of the $\Delta$ Dalitz decay $\left(\Delta \rightarrow e^{+} e^{-} N\right)$ measured at HADES. The comparison of our calculations with the HADES $\Delta$ (1232) Dalitz decay cross-sections can be found in [38]. Those results are also discussed in the presentations of B. Ramstain and P. Salabura [39, 40].

The present discussion of the $\gamma^{*} N \rightarrow \Delta(1232)$ transition is simplified because we ignore the contributions of the $\Delta(1232) d$-wave states $[11,12,41,42]$. The procedure is justified because the admixture terms are of the order of $0.7 \%$ for both states. Those states are, however, important for the sub-leading transition form factors, the electric $\left(G_{E}^{*}\right)$ and Coulomb $\left(G_{C}^{*}\right)$ quadrupole form factors. Those form factors are described in the Sect. 4, at low $Q^{2}$.

\subsection{Radial excitations}

The covariant spectator quark model can also be applied to the radial excitations of the nucleon. The first radial excitation, $N(1440)$ or Roper, can be defined as a system which differs from the nucleon only by the radial structure, and has a zero in the radial wave function [43, 44]. The $N(1440)$ state can then be determined defining a radial wave function $\psi_{R}$ which is orthogonal to the nucleon and has a single node. The transition form factors are then calculated without any adjustable parameters. The results (solid line) are presented in Fig. 2 in comparison with the CLAS data [45-47].

From the graphs, we conclude that the model describe well the data above $1.5 \mathrm{GeV}^{2}$ for both form factors. Those results are an indication that $N(1440)$ is in fact the first radial excitation of the nucleon. The disagreement at low $Q^{2}$ can be a consequence of not taking into account possible meson cloud effects [43, 44].

The $\gamma^{*} N \rightarrow N(1440)$ form factors have been also estimated within the AdS/QCD framework [48, 49]. In the leading twist approximation, where the baryons correspond to three-quark systems, the nucleon and the Roper transition form factors depend on three independent couplings, which can be fixed by the nucleon elastic form factor data, within certain limits. The results for the Roper are then determined without any additional fit. Those are also represented in Fig. 2 by the red band between the upper and lower limits. The results of the Pauli form factor $F_{2}^{*}$ are in excellent agreement with the data. Overall, it is interesting to observe that the two estimates converge to the same result for large values of $Q^{2}$, which can be seen as a consequence of the dominance of the valence quark degrees of freedom at large $Q^{2}$. 

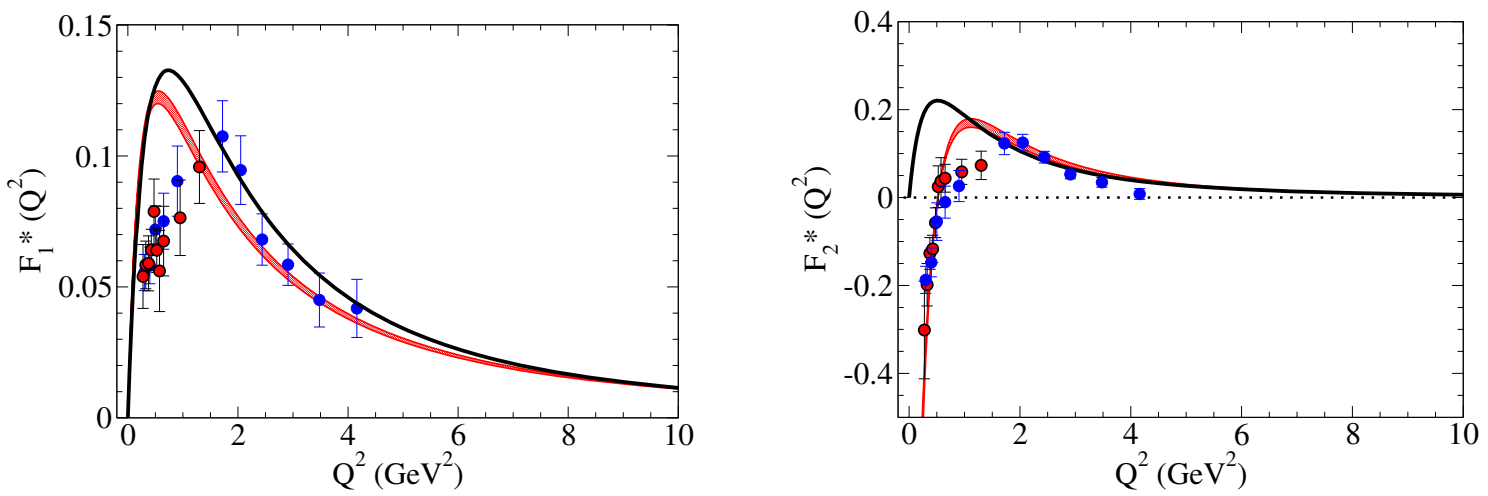

Figure 2. $\gamma^{*} N \rightarrow N(1440)$ transition form factors. The solid line represent the estimate of the covariant quark model [43]. The red band represent the estimated from AdS/QCD taking into account the uncertainty of the couplings [48]. Data from CLAS [45-47].
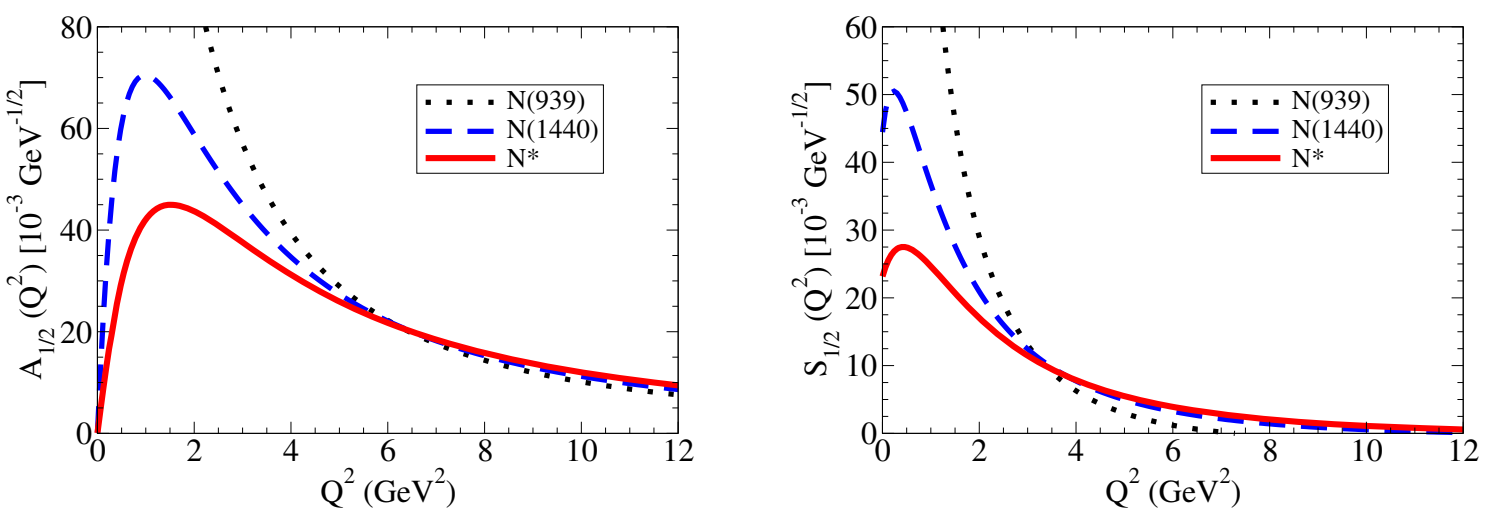

Figure 3. $\gamma^{*} N \rightarrow N^{*}$ transition amplitudes for the first and the second $\left(N^{*}\right)$ radial excitations of the nucleon. $N(1930)$ represent the nucleon's equivalent amplitudes (description in the main text).

The covariant spectator quark model can also be used to estimate the second radial excitation of the nucleon. In a previous work [44], we assumed tentatively that the state could be the $N(1710) \frac{1}{2}^{+}$, but the assumption was discarded by recent measurements from CLAS [50]. The next candidate is then the state $N(1880) \frac{1}{2}^{+}$. In this case, we compare the results directly to the helicity amplitudes $A_{1 / 2} \propto\left(F_{1 p}^{*}+F_{2 p}^{*}\right)$ and $S_{1 / 2} \propto\left(F_{1 p}^{*}-\tau F_{2 p}^{*}\right)$, where $\tau=\frac{Q^{2}}{\left(M_{N}+M_{R}\right)^{2}}$. The results for the Roper and the next radial excitation $\left(N^{*}\right)$ are presented in Fig. 3. In the graphs, we include also the equivalent amplitudes of the nucleon $A_{1 / 2} \propto G_{M}$ and $S_{1 / 2} \propto G_{E}$ (the conversion factors are calculated using the mass of the Roper) [44]. Note that, the helicity amplitude representation is particularly useful, because it emphasizes the similarity of the amplitudes associated with the different states at large $Q^{2}$, particularly to $A_{1 / 2}$. The corollary of this result is that, one can use the experimental results of the magnetic form factor of the proton, $G_{M p}$, to predict the amplitude $A_{1 / 2}$ for the Roper and $N(1880) \frac{1}{2}^{+}$states in the range $Q^{2}=10-30 \mathrm{GeV}^{2}$. Hopefully our predictions for $A_{1 / 2} \propto G_{M p}$ can be tested in a near future.

Similarly to the case of the nucleon, we can use the formalism to estimate the first radial excitation of the $\Delta(1232)$, the state $\Delta(1600)$, based on our model for the $\Delta(1232)$. Our estimates are valid for large $Q^{2}$ and dif- fer from the present measurements at $Q^{2}=0$, although the estimates approach the experimental data when we take into account the meson cloud dressing based on a simplified model derived from the $\gamma^{*} N \rightarrow \Delta(1232)$ pion cloud parametrization [13]. Our estimates for the $\gamma^{*} N \rightarrow$ $\Delta(1600)$ form factors may be tested in a near future, since data are under analysis and results are expected soon [51].

\section{$3.3 \gamma^{*} N \rightarrow N(1520)$ and $\gamma^{*} N \rightarrow N(1535)$ transitions}

The $N(1520)$ and $N(1535)$ are negative parity states. Applications of the covariant spectator quark model to the $\gamma^{*} N \rightarrow N(1535)$ and $\gamma^{*} N \rightarrow N(1520)$ transitions are discussed in Refs. [14, 15, 24] based on different prescriptions to the radial wave functions.

In particular the $\gamma^{*} N \rightarrow N(1520)$ transition was extended to the timelike region [15] and to the study of the HADES N(1520) Dalitz decays. Some preliminary results are discussed in the B. Ramstein presentation [39].

Recently the $\gamma^{*} N \rightarrow N(1535)$ and $\gamma^{*} N \rightarrow N(1520)$ transitions have been studied within the semirelativistic approximation. The semirelativistic approximation is characterized by the following properties [22]: i) the mass difference $\left(M_{R}\right.$ and $\left.M_{N}\right)$ is neglected in a first approximation; ii) the radial wave function of the resonance $\psi_{R}$ has the same form as the nucleon radial wave function. 

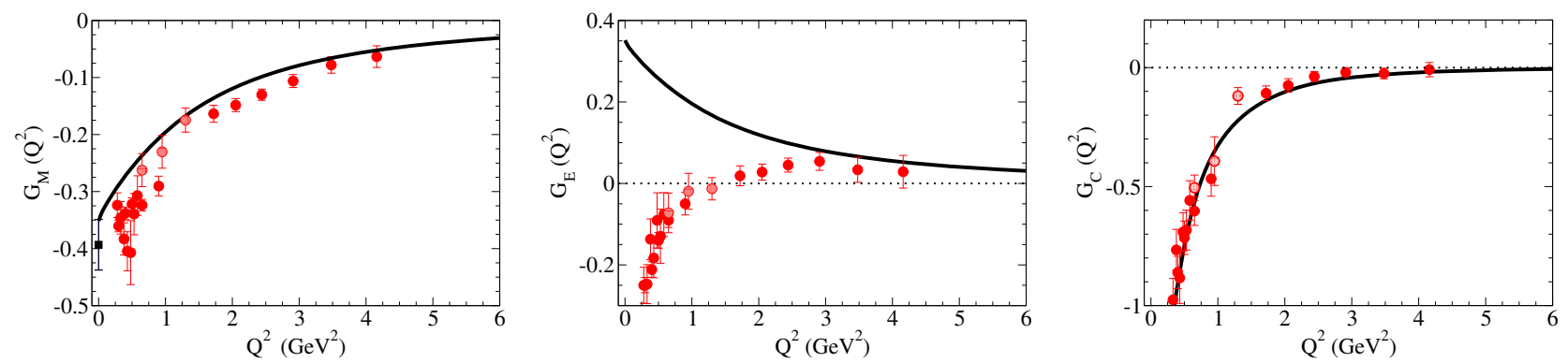

Figure 4. $\gamma^{*} N \rightarrow N(1520)$ transition form factors. The solid line is the result of the semirelativistic approximation. Data from JLab (circles) [6, 45, 47] and PDG (square) [6].
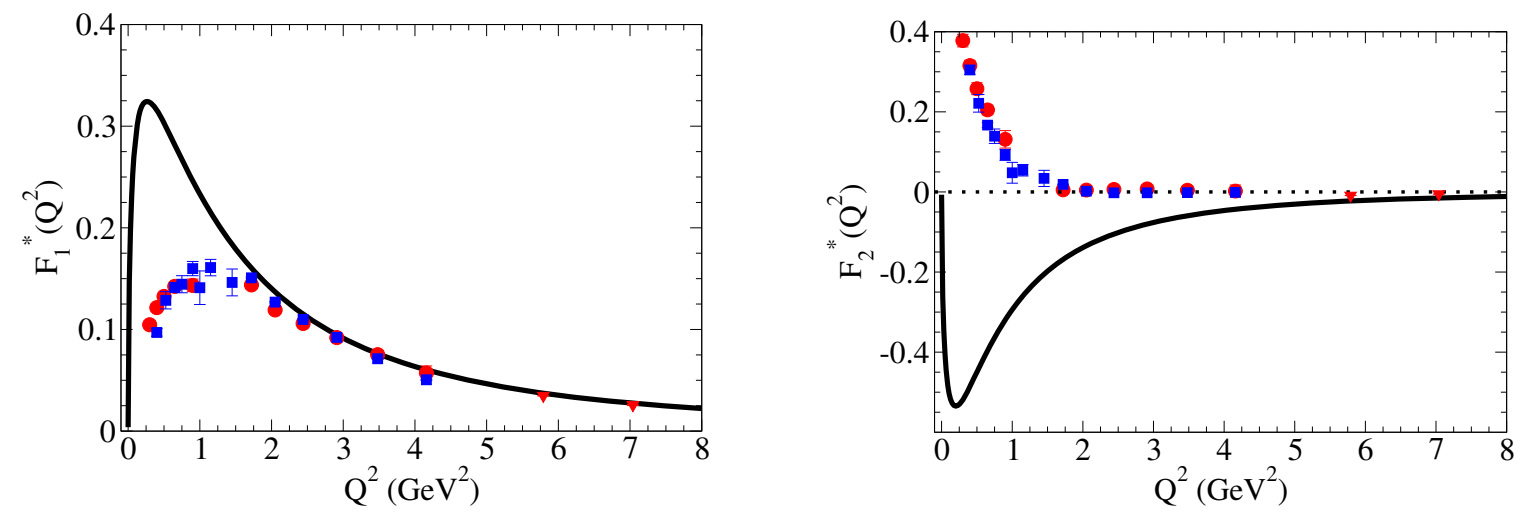

Figure 5. $\gamma^{*} N \rightarrow N(1535)$ Dirac transition form factor. Data from JLab (circles and triangles) [6, 45, 54] and MAID (squares) [3].

Under these conditions the orthogonality between the states is naturally ensured, and the transition form factors are calculated without the introduction of any additional parameters [22]. Those estimates are therefore true predictions. All model parameters are determined by the original parametrization of the nucleon system. The semirelativistic estimates for the $\gamma^{*} N \rightarrow N(1520)$ and $\gamma^{*} N \rightarrow N(1535)$ form factors are presented in Figs. 4 and 5, respectively.

In Fig. 4 we compare our estimates for the $\gamma^{*} N \rightarrow$ $N(1520)$ transition form factors: $G_{M}$ (magnetic), $G_{E}$ (electric) and $G_{C}$ (Coulomb) with the available data. One can notice that one obtain a good description of the data for $G_{M}$ and $G_{C}$, particularly for $Q^{2}>1 \mathrm{GeV}^{2}$. As for $G_{E}$ the estimate fails for $Q^{2}<2.5 \mathrm{GeV}^{2}$, which seems to be a consequence of the omission of the meson cloud effects. In the context of the covariant spectator quark model the result for $G_{E}$ is a consequence of the estimate $A_{3 / 2} \equiv 0$, indicating that the amplitude $A_{3 / 2}$ is dominated by meson cloud effects [14, 15].

In Fig. 5, we compare the estimates of the Dirac $\left(F_{1}^{*}\right)$ and Pauli $\left(F_{2}^{*}\right) \gamma^{*} N \rightarrow N(1535)$ with the available data. From the graph for $F_{1}^{*}$, we conclude that the model describe well the data above $1.5 \mathrm{GeV}^{2}$. From the graph for $F_{2}^{*}$, we can notice that the model fails completely the description of the data (wrong sign). These two discrepancies may be an indication of the impact of the meson cloud effects, not included in the present calculations. There are evidences that the inclusion of meson cloud effects can in fact improve the description of the data $[16,17,23,24,52,53]$.
The results for $F_{2}^{*}$ in particular raises the possibility that there are cancellations between the valence quark effects (estimated by our model) and the meson cloud effects (not included in our estimate). In that case, we can explain the experimental result $F_{2}^{*} \approx 0$ for $Q^{2}>1.5$ $\mathrm{GeV}^{2}$. The implication of the previous result is that the $\gamma^{*} N \rightarrow N(1535)$ amplitudes are related by [52]

$$
S_{1 / 2}=-\frac{\sqrt{1+\tau}}{\sqrt{2}} \frac{M_{R}^{2}-M_{N}^{2}}{2 M_{R} Q} A_{1 / 2},
$$

for $Q^{2}>1.5 \mathrm{GeV}^{2}$, where $\tau=\frac{Q^{2}}{\left(M_{R}+M_{N}\right)^{2}}$ and $Q=\sqrt{Q^{2}}$.

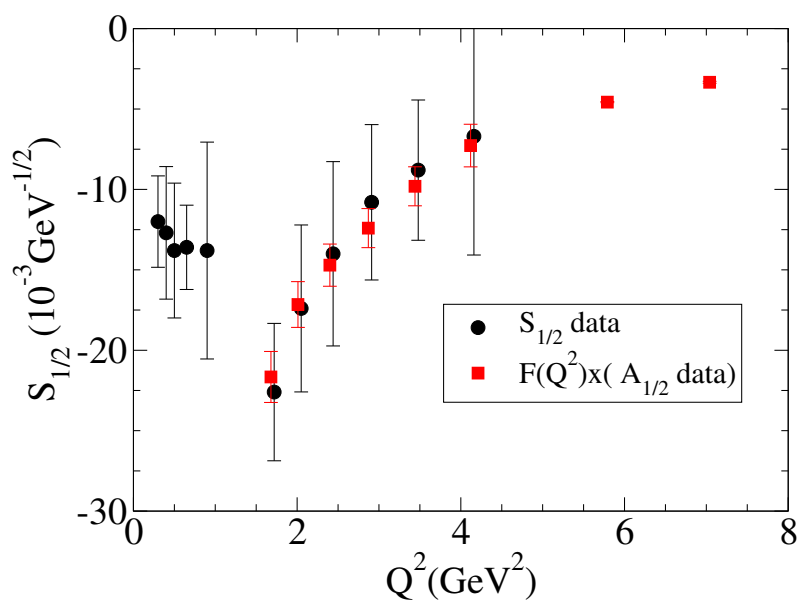

Figure 6. $\gamma^{*} N \rightarrow N(1535)$ amplitude $S_{1 / 2}$. Data compared with estimate from Eq. (3). Data from CLAS [45, 54]. 

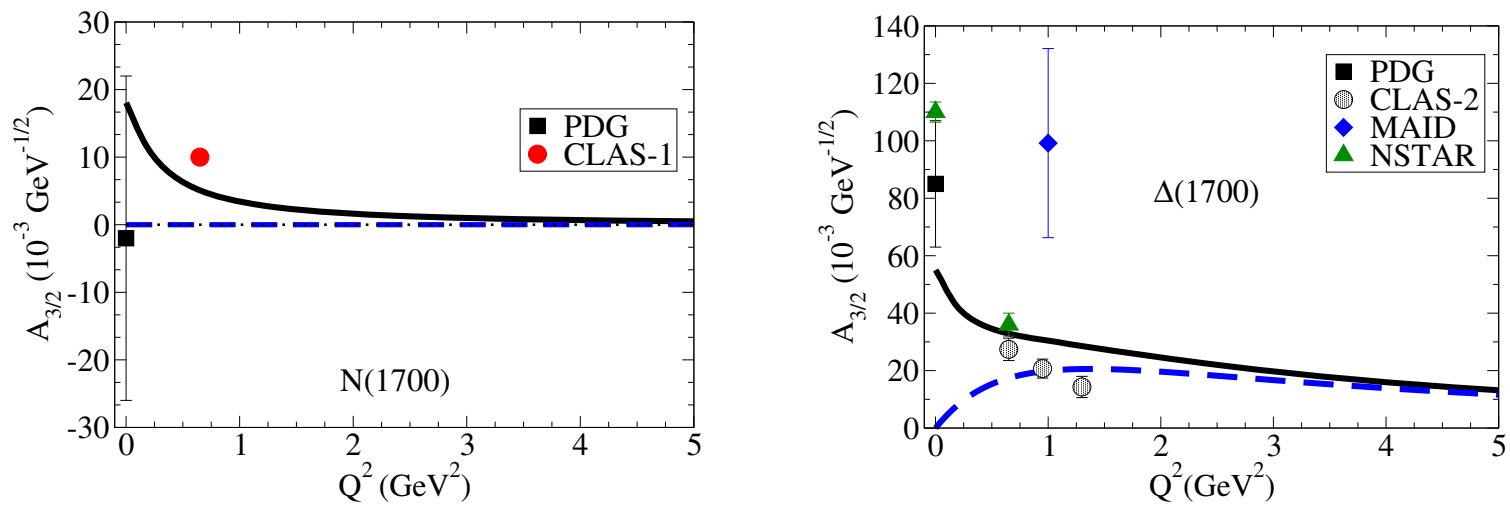

Figure 7. Examples of estimates of amplitudes associated with negative parity states based on the single quark transition model [23]. The solid line represent a model where the meson cloud effects are taken into account. Check [23] for the description of the data.

The relation (3) is tested in Fig. 6 based on the data for the amplitude $A_{1 / 2}$. The last two point present a prediction for the $S_{1 / 2}$ data. New large $Q^{2}$ data are needed in order to test the relation (3) for higher values of $Q^{2}$.

The results for the $\gamma^{*} N \rightarrow N(1535)$ transition motivated the use of the valence quark model to calibrate the meson cloud contributions and the extension of the results to the timelike region and the $N(1535)$ Dalitz decay [17].

Overall, we can conclude that the semirelativistic approximation provides a good description of the $\gamma^{*} N \rightarrow$ $N(1520)$ and $\gamma^{*} N \rightarrow N(1535)$ transition form factors for $Q^{2}>1.5 \mathrm{GeV}^{2}$. The exceptions are the Pauli form factor for $N(1535)$ and the electric form factor for $N(1520)$, the two cases for which there are indications that meson cloud effects may be more significant. We emphasize that all the calculations include no free parameters, apart the calibration of the nucleon system. A more detailed discussion of the results for the $\gamma^{*} N \rightarrow N(1535)$ and $\gamma^{*} N \rightarrow N(1520)$ transitions can be found in [14, 16, 17, 23, 24, 52, 53].

\subsection{Single Quark Transition Model}

The covariant spectator quark model can also be used in combination with single quark transition model (SQTM) [23, 55], since the two models are based on similar principles: electromagnetic interaction in impulse approximation and wave functions determined by the $S U(6) \otimes O(3)$ structure [9].

Of particular interest is the $\left[70,1^{-}\right]$supermultiplet, whose transverse amplitudes, $A_{1 / 2}$ and $A_{3 / 2}$, can be determined by three independent functions of $Q^{2}: A, B$ and $C$, according with the SQTM. In these circumstances, one can determine the transverse amplitudes of the states $N(1650) \frac{1}{2}^{-}, \Delta(1620) \frac{1}{2}^{-}, N(1700) \frac{3}{2}^{-}$and $\Delta(1700) \frac{3}{2}^{-}$, once the functions $A, B$ and $C$ are known. These three functions can be calculated using the covariant spectator quark models for the $N(1520) \frac{3}{2}^{-}$and $N(1535) \frac{1}{2}^{-}$transverse amplitudes [5, 14, 23, 24].

The estimates for the $N(1650) \frac{1}{2}^{-}$and $\Delta(1620) \frac{1}{2}^{-}$amplitudes are consistent with available data $[3,6]$ for large $Q^{2}$ [23]. For the remaining cases, there are no available data for $Q^{2}>2 \mathrm{GeV}^{2}$. As an example, we present in Fig. 7, the results for the amplitude $A_{3 / 2}$ associated with the states $N(1700)$ and $\Delta(1700)$. Note in both cases the lack of data for $Q^{2}>1.5 \mathrm{GeV}^{2}$. Our estimates can be tested, then only when new large $Q^{2}$ data from the JLab$12 \mathrm{GeV}$ upgrade became available [1].

\section{Transition form factors at low $Q^{2}$}

One can also use the covariant spectator quark model to estimate transition form factors at low $Q^{2}$. In general the estimates cannot be compared directly with the data because the meson cloud contributions can be significant and camouflage the valence quark contributions.

In the case of the $\gamma^{*} N \rightarrow \Delta(1232)$ there is the possibility of estimating the valence quark effects since there are lattice QCD data available [36]. We obtain nonzero contributions to the electric $\left(G_{E}\right)$ and Coulomb $\left(G_{C}\right)$ quadrupole form factors when we consider $d$-wave contributions to the $\Delta(1232)$ wave function $[11,12,41,42]$. The contributions of those states for the transition form factors are small (about one order of magnitude) when compared with the experimental data, but their magnitude is comparable with the lattice QCD data $[11,12]$. One can then use lattice QCD data to estimate the free parameters of the quark model performing fits to the data $[12,21]$. Once extrapolated to the physical limit, one obtains the valence quark contributions to the quadrupole form factors [12].

The experimental data for $G_{E}$ and $G_{C}$ are represented in Fig. 8. For convenience of the discussion the results for $G_{C}$ are multiplied by $\kappa=\frac{M_{\Delta}-M_{N}}{2 M_{\Delta}}$. The valence quark contributions discussed above are represented by the thin lines (solid line for $G_{E}$ and dashed line for $\kappa G_{C}$ ). Notice, in particular, the difference of magnitude in comparison with the final results (thick lines). The thick lines are discussed next.

To describe the physical data, we need to take into account the pion cloud contributions to the electric and Coulomb quadrupole form factors. There are in the literature estimates of the pion cloud contributions to the quadrupole form factors based on the large- $N_{c}$ limit [56, 57]. Those estimates are, however, inconsistent with the long wavelength limit also known as Siegert's theorem, which states that in the limit $Q^{2}=-\left(M_{\Delta}-M_{N}\right)^{2}$, the two 

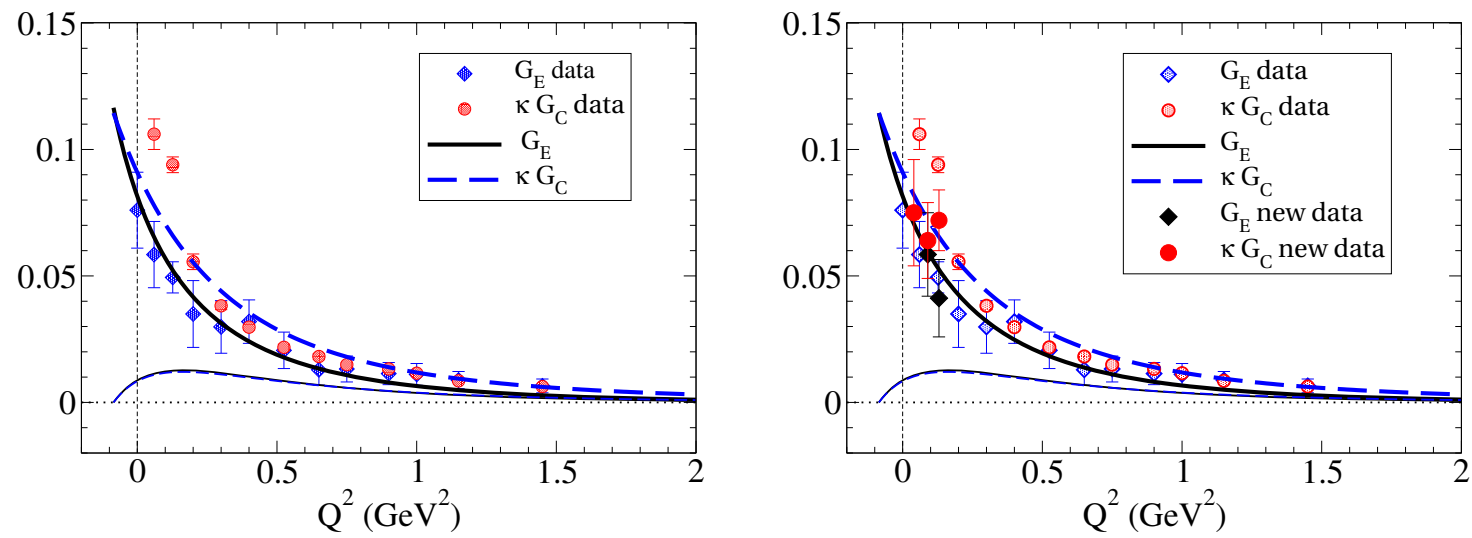

Figure 8. $\gamma^{*} N \rightarrow \Delta(1232)$ quadrupole form factors. The Coulomb form factor is normalized by $\kappa=\frac{M_{\Delta}-M_{N}}{2 M_{\Delta}}$ for convenience. The model combines valence quark and pion cloud contributions. Data from [6,64]. Left: Estimate with pion cloud contribution from [62]. Right: Estimate with pion cloud contribution from [63]. New low- $Q^{2}$ data from [66].

quadrupole form factors are related by [37, 58-65]

$$
G_{E}=\frac{M_{\Delta}-M_{N}}{2 M_{\Delta}} G_{C}
$$

The point $Q^{2}=-\left(M_{\Delta}-M_{N}\right)^{2}$ is also known as pseudothreshold.

The original large- $N_{c}$ estimates of the pion cloud contributions [56, 57, 62], can be improved with a correction of the order $1 / N_{c}^{2}$ in $G_{E}$ in order to be consistent with Siegert's theorem (4) [62-64]. Combining the valence quark contributions with an improved large- $N_{c}$ estimate of the pion cloud for $G_{E}$ [62], we obtain a good description of the quadrupole form factor data. This can be observed in the left panel of the Fig. 8. The parametrization of [62] violates Siegert's theorem only a term in $1 / N_{c}^{4}$. The estimate fails only for the first two points for $G_{C}$.

On the right panel of Fig. 8, we present a new calculation where the pion cloud parametrization is further improved in order to satisfy (4) exactly. In the graph, the low- $Q^{2}$ data for $G_{E}$ and $G_{C}$ are replaced by new data which corrects the previous analysis [66]. The new estimate is in perfect agreement with the new data for both form factors.

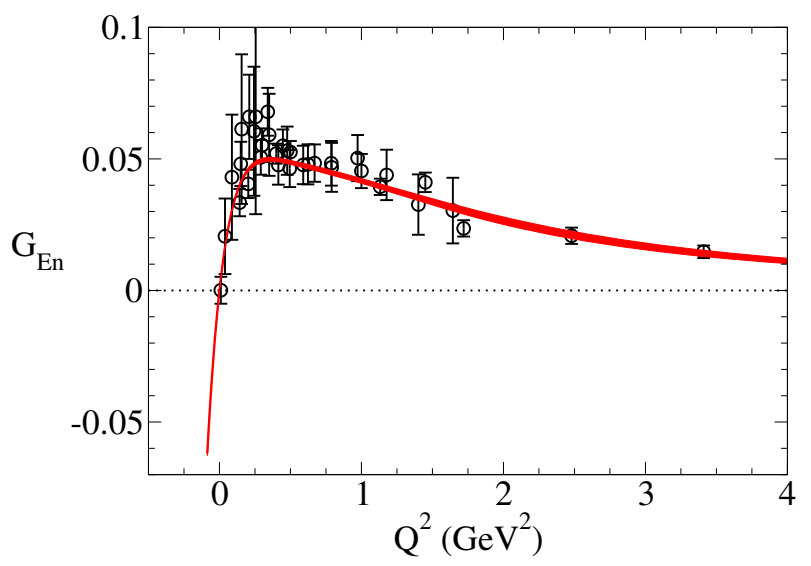

Figure 9. Neutron electric form factor $G_{E n}$ determined by a global fit to the $G_{E n}$ and $G_{E}$ and $G_{C}$ quadrupole form factors [64].
These results, demonstrate that the $\gamma^{*} N \rightarrow \Delta(1232)$ quadrupole form factors can be completely described by a combination of valence quark and pion cloud contributions. The pion cloud parametrizations include no free parameters, and the valence quark contributions are determined exclusively by the lattice QCD data [12]. There are, therefore, no fits to the physical data in the estimates from Fig. 8.

The pion cloud parametrizations for $G_{E}$ and $G_{C}$ are based on relations $G_{E} \propto G_{E n}$ and $G_{C} \propto G_{E n}$, valid at low $Q^{2}[56,57,62,63]$, where $G_{E n}$ is the neutron electric form factor. Inspired by the previous results, we use the relation between the $\gamma^{*} N \rightarrow \Delta(1232) G_{E}$ and $G_{C}$ form factors and $G_{E n}$ to obtain a more accurate parametrization of $G_{E n}$ [64]. Our final parametrization for $G_{E n}$ is presented in Fig. 9. Also estimated are the second moment of the neutron electric form factor: $r_{4}^{4} \simeq-0.4 \mathrm{fm}^{4}$, and the electric and Coulomb square radii: $r_{E 2}^{2}=2.2 \pm 0.2 \mathrm{fm}^{2}$ and $r_{C 2}^{2}=1.8 \pm 0.1 \mathrm{fm}^{2}$.

\section{Summary and Conclusions}

We present covariant estimates of the $\gamma^{*} N \rightarrow N^{*}$ transition form factors for large $Q^{2}$ for the $N^{*}$ states: $\Delta(1232), N(1440), N(1520), N(1535), N(1880), N(1700)$ and $\Delta(1700)$. There are also estimates for the states $N(1650), \Delta(1600)$ and $\Delta(1620)$, not discussed in detail due to the lack of data for $Q^{2}>2 \mathrm{GeV}^{2}$.

Our estimates are valid in principle for $Q^{2}>2 \mathrm{GeV}^{2}$, since the valence quark effects are expected to dominate over the meson cloud effects when $Q^{2}$ increases. In some cases, however, we conclude that the valence quark contributions are insufficient to explain the data in the range $Q^{2}=1-4 \mathrm{GeV}^{2}$, implying that the meson cloud effects may be significant in that range. Examples of those cases are the $\gamma^{*} N \rightarrow N(1535)$ Pauli form factor and the $\gamma^{*} N \rightarrow$ $\Delta(1232)$ quadrupole form factors.

Our estimates are based mainly on the calibrations for the nucleon and $\Delta(1232)$ systems. Future large $Q^{2}$ data, such as the data from the JLab- $12 \mathrm{GeV}$ upgrade will be fundamental to test our estimates at large $Q^{2}$, or to refine 
our calibrations, if the new data for the nucleon and the $\Delta(1232)$ deviates from the present trend.

Future data at low $Q^{2}$, particularly in the range $Q^{2}=0-0.3 \mathrm{GeV}^{2}$, will be also very important to establish the shape of the transition form factors near $Q^{2}=0$. The $N^{*}$ contributions to the nucleon Compton scattering are particularly sensitive to those form factors [67].

\section{Acknowledgments}

G. R. was supported by the Fundação de Amparo à Pesquisa do Estado de São Paulo (FAPESP): Project No. 2017/02684-5, Grant No. 2017/17020-BCO-JP.

\section{References}

[1] I. G. Aznauryan et al., Int. J. Mod. Phys. E 22, 1330015 (2013).

[2] I. G. Aznauryan and V. D. Burkert, Prog. Part. Nucl. Phys. 67, 1 (2012).

[3] D. Drechsel, S. S. Kamalov and L. Tiator, Eur. Phys. J. A 34, 69 (2007).

[4] V. D. Burkert and T. S. H. Lee, Int. J. Mod. Phys. E 13, 1035 (2004).

[5] G. Ramalho, Few Body Syst. 59, 92 (2018).

[6] V. I. Mokeev, https://userweb.jlab.org/ mokeev/ resonance_electrocouplings/

[7] F. Gross, G. Ramalho and M. T. Peña, Phys. Rev. C 77, 015202 (2008).

[8] G. Ramalho, K. Tsushima and F. Gross, Phys. Rev. D 80, 033004 (2009).

[9] S. Capstick and W. Roberts, Prog. Part. Nucl. Phys. 45, S241 (2000).

[10] G. Ramalho, M. T. Peña and F. Gross, Eur. Phys. J. A 36, 329 (2008).

[11] G. Ramalho, M. T. Peña and F. Gross, Phys. Rev. D 78, 114017 (2008).

[12] G. Ramalho and M. T. Peña, Phys. Rev. D 80, 013008 (2009).

[13] G. Ramalho and K. Tsushima, Phys. Rev. D 82, 073007 (2010).

[14] G. Ramalho and M. T. Peña, Phys. Rev. D 89, 094016 (2014).

[15] G. Ramalho and M. T. Peña, Phys. Rev. D 95, 014003 (2017).

[16] G. Ramalho, D. Jido and K. Tsushima, Phys. Rev. D 85, 093014 (2012).

[17] G. Ramalho and M. T. Peña, Phys. Rev. D 101, 114008 (2020).

[18] G. Ramalho and K. Tsushima, Phys. Rev. D 87, 093011 (2013).

[19] G. Ramalho and K. Tsushima, Phys. Rev. D 88, 053002 (2013).

[20] F. Gross, G. Ramalho and M. T. Peña, Phys. Rev. D 85, 093005 (2012).

[21] G. Ramalho and M. T. Peña, J. Phys. G 36, 115011 (2009).

[22] G. Ramalho, Phys. Rev. D 95, 054008 (2017).
[23] G. Ramalho, Phys. Rev. D 90, 033010 (2014).

[24] G. Ramalho and M. T. Peña, Phys. Rev. D 84, 033007 (2011).

[25] G. Ramalho, K. Tsushima and A. W. Thomas, J. Phys. G 40, 015102 (2013).

[26] G. Ramalho, J. P. B. C. De Melo and K. Tsushima, Phys. Rev. D 100, 014030 (2019).

[27] G. Ramalho and M. T. Peña, Phys. Rev. D 85, 113014 (2012).

[28] G. Ramalho, M. T. Peña, J. Weil, H. van Hees and U. Mosel, Phys. Rev. D 93, 033004 (2016).

[29] S. S. Kamalov and S. N. Yang, Phys. Rev. Lett. 83, 4494 (1999).

[30] T. Sato and T. S. H. Lee, Phys. Rev. C 63, 055201 (2001).

[31] B. Julia-Diaz, H. Kamano, T.-S. H. Lee, A. Matsuyama, T. Sato and N. Suzuki, Phys. Rev. C 80, 025207 (2009).

[32] D. Ronchen et al., Eur. Phys. J. A 49, 44 (2013).

[33] H. W. Hammer, D. Drechsel and U. G. Meissner, Phys. Lett. B 586, 291 (2004).

[34] U. G. Meissner, AIP Conf. Proc. 904, 142 (2007).

[35] V. Pascalutsa, M. Vanderhaeghen and S. N. Yang, Phys. Rept. 437, 125 (2007).

[36] C. Alexandrou, G. Koutsou, H. Neff, J. W. Negele, W. Schroers and A. Tsapalis, Phys. Rev. D 77, 085012 (2008).

[37] H. F. Jones and M. D. Scadron, Annals Phys. 81, 1 (1973).

[38] J. Adamczewski-Musch et al. [HADES Collaboration], Phys. Rev. C 95, 065205 (2017).

[39] B. Ramstein, these proceedings.

[40] P. Salabura, these proceedings.

[41] G. Ramalho, M. T. Peña and F. Gross, Phys. Rev. D 81, 113011 (2010).

[42] G. Ramalho, M. T. Peña and A. Stadler, Phys. Rev. D 86, 093022 (2012).

[43] G. Ramalho and K. Tsushima, Phys. Rev. D 81, 074020 (2010).

[44] G. Ramalho and K. Tsushima, Phys. Rev. D 89, 073010 (2014).

[45] I. G. Aznauryan et al. [CLAS Collaboration], Phys. Rev. C 80, 055203 (2009).

[46] V. I. Mokeev et al. [CLAS Collaboration], Phys. Rev. C 86, 035203 (2012).

[47] V. I. Mokeev et al., Phys. Rev. C 93, 025206 (2016).

[48] G. Ramalho and D. Melnikov, Phys. Rev. D 97, 034037 (2018).

[49] G. Ramalho, Phys. Rev. D 96, 054021 (2017).

[50] K. Park et al. [CLAS Collaboration], Phys. Rev. C 91, 045203 (2015).

[51] R. Gothe, these proceedings.

[52] G. Ramalho and K. Tsushima, Phys. Rev. D 84, 051301 (2011).

[53] D. Jido, M. Doering and E. Oset, Phys. Rev. C 77, 065207 (2008).

[54] M. M. Dalton et al., Phys. Rev. C 80, 015205 (2009). 
[55] V. D. Burkert, R. De Vita, M. Battaglieri, M. Ripani and V. Mokeev, Phys. Rev. C 67, 035204 (2003).

[56] V. Pascalutsa and M. Vanderhaeghen, Phys. Rev. D 76, 111501 (2007).

[57] A. J. Buchmann, Can. J. Phys. 87, 773 (2009).

[58] R. C. E. Devenish, T. S. Eisenschitz and J. G. Korner, Phys. Rev. D 14, 3063 (1976).

[59] A. J. Buchmann, U. Meyer, A. Faessler and E. Hernandez, Phys. Rev. C 58, 2478 (1998).
[60] G. Ramalho, Phys. Lett. B 759, 126 (2016).

[61] G. Ramalho, Phys. Rev. D 93, 113012 (2016).

[62] G. Ramalho, Phys. Rev. D 94, 114001 (2016).

[63] G. Ramalho, Eur. Phys. J. A 54, 75 (2018).

[64] G. Ramalho, Eur. Phys. J. A 55, 32 (2019).

[65] G. Ramalho, Phys. Rev. D 100, 114014 (2019).

[66] A. Blomberg et al., Phys. Lett. B 760, 267 (2016).

[67] G. Eichmann and G. Ramalho, Phys. Rev. D 98, 093007 (2018). 